January 2022

\title{
Pivoting to be Patient-Centered: A Study on Hidden Psychological Costs of Tele-Healthcare and the Patient-Provider Relationship
}

\author{
Adam Neufeld \\ University of Calgary, adam.neufeld@ucalgary.ca \\ Vishal Bhella \\ University of Calgary, vishal.bhella@ucalgary.ca \\ Clark Svrcek \\ University of Calgary, clark.svrcek@ucalgary.ca
}

Follow this and additional works at: https://nsuworks.nova.edu/ijahsp

Part of the Medical Humanities Commons, and the Telemedicine Commons

\section{Recommended Citation}

Neufeld A, Bhella V, Svrcek C. Pivoting to be Patient-Centered: A Study on Hidden Psychological Costs of Tele-Healthcare and the Patient-Provider Relationship. The Internet Journal of Allied Health Sciences and Practice. 2022 Jan 03;20(1), Article 8.

This Manuscript is brought to you for free and open access by the College of Health Care Sciences at NSUWorks. It has been accepted for inclusion in Internet Journal of Allied Health Sciences and Practice by an authorized editor of NSUWorks. For more information, please contact nsuworks@nova.edu. 


\title{
Pivoting to be Patient-Centered: A Study on Hidden Psychological Costs of Tele- Healthcare and the Patient-Provider Relationship
}

\begin{abstract}
Purpose: While the COVID-19 pandemic has necessitated a shift toward virtual medicine, there are some potentially important limitations with this modality of healthcare. One such concern, which has not yet been elucidated, is how telephone-based versus in-person visits differentially impact patients' perceived autonomy and the resulting patient-provider relationship. Grounded in self-determination theory (SDT), this pilot study addresses this question by investigating the association between patients' perceived autonomy support and relationship needs satisfaction (autonomy, competence, relatedness) in both types of visits with their family doctor and care team. Methods: Running from Sept. 2020 to Feb. 2021, data was collected via convenience sampling from $n=66$ patients (34 in person, 32 virtual) nested within $k=6$ family physicians. Patients completed an online survey containing two previously validated scales derived from SDT: The Healthcare Climate Questionnaire and the Basic Need Satisfaction in Relationships Scale. Each scale was adapted to reflect a virtual or in-person visit experience. A random effects model captured the relationship between the motivational variables in each group, adjusting for various sociodemographic effects. Results: Both groups' perceived autonomy support positively related to their relationship needs satisfaction with their family doctor and care team. Compared to traditional in-person visits, patients perceived the virtual healthcare climate as significantly less autonomy-supportive. Conclusions: In line with SDT, findings from this study suggest that when patients sense a more autonomy-supportive healthcare climate, they will experience a more needs-satisfying relationship with their family doctor and healthcare team. These results have potentially significant implications for supporting patient motivation and facilitating optimal health and wellness outcomes, particularly within the virtual care environment.
\end{abstract}




\title{
IIJAHSP \\ The Internet Journal of Allied Health Sciences and Practice \\ Dedicated to allied health professional practice and education \\ Vol. 20 No. 1 ISSN 1540-580X
}

\section{Pivoting to be Patient-Centered: A Study on Hidden Psychological Costs of Tele-Healthcare and the Patient-Provider Relationship}

\author{
Adam Neufeld \\ Vishal Bhella \\ Clark Svrcek \\ University of Calgary \\ Canada
}

\begin{abstract}
Purpose: While the COVID-19 pandemic has necessitated a shift toward virtual medicine, there are some potentially important limitations with this modality of healthcare. One such concern, which has not yet been elucidated, is how telephone-based versus in-person visits differentially impact patients' perceived autonomy and the resulting patient-provider relationship. Grounded in selfdetermination theory (SDT), this pilot study addresses this question by investigating the association between patients' perceived autonomy support and relationship needs satisfaction (autonomy, competence, relatedness) in both types of visits with their family doctor and care team. Methods: Running from Sept. 2020 to Feb. 2021, data was collected via convenience sampling from $n=$ 66 patients (34 in person, 32 virtual) nested within $\mathrm{k}=6$ family physicians. Patients completed an online survey containing two previously validated scales derived from SDT: The Healthcare Climate Questionnaire and the Basic Need Satisfaction in Relationships Scale. Each scale was adapted to reflect a virtual or in-person visit experience. A random effects model captured the relationship between the motivational variables in each group, adjusting for various sociodemographic effects. Results: Both groups' perceived autonomy support positively related to their relationship needs satisfaction with their family doctor and care team. Compared to traditional in-person visits, patients perceived the virtual healthcare climate as significantly less autonomysupportive. Conclusions: In line with SDT, findings from this study suggest that when patients sense a more autonomy-supportive healthcare climate, they will experience a more needs-satisfying relationship with their family doctor and healthcare team. These results have potentially significant implications for supporting patient motivation and facilitating optimal health and wellness outcomes, particularly within the virtual care environment.
\end{abstract}

Keywords: tele-medicine, COVID-19, perceived autonomy, patient-provider relationship 
"Wherever the art of Medicine is loved, there is also a love of humanity" - Hippocrates

\section{INTRODUCTION}

Ever since the coronavirus 2019 (COVID-19) pandemic struck, healthcare workers across the world have had to quickly shift to virtual (e.g., telephone and videoconference) methods of patient care, to safely connect with and serve their patients. ${ }^{1,2,3}$ Unsurprisingly, studies show this effort has been vital in reducing the mental health burden associated with COVID-19.4 Virtual healthcare also affords patients the convenience, comfort, and safety of medical appointments from their own home, while facilitating various medical needs: medication optimizing and prescription refills, screening labs and investigations (e.g., blood work, imaging), and health-related conversations (i.e., for acute and chronic physical, psychological, and social concerns). Some studies suggest that patients and physicians may even prefer virtual visits, if not for concerns about technical glitches, diminished information privacy, and the inability to conduct physical exams. 5,6,7

Of course, most physicians will agree that it is difficult to manage various physical (e.g., abdominal pain, skin lesions, shortness of breath) and mental (e.g., anxiety, depression, addictions) health issues over the telephone.8.,9,10 Beyond this limitation, however, there is also a prevailing sentiment that healthcare providers and patients seem to be conveying, which is that virtual medicine is simply not as humanistic or satisfying, and that it threatens the healing bond between patient and provider. ${ }^{11,12} \mathrm{In}$ an attempt to address this issue, some have even begun to adapt existing frameworks in primary care, particularly during the COVID-19 pandemic. ${ }^{13}$ Still yet, the motivational impact of this shift - from touch to talk in medicine - remains unclear. ${ }^{2,14}$ From our vantage point as family physicians, while societal pressures to adopt virtual modalities of health care are abundant, lived experiences and empirical clues suggest that pressing forward in this direction may come with hidden psychological costs - not just to patients or providers, but to the patient-provider relationship. The question is: in what meaningful ways will virtual care change this relationship, and to what extent? Answering these questions requires a better macro-level understanding of the motivational-psychological effect of virtual health care visits.

Self-determination theory (SDT) represents a broad framework for the study of human motivation, development, and well-being. ${ }^{15}$ With over forty years of evidential support across different domains and cultures (e.g., healthcare, education, organizations, sports), its model is well-suited to address the above research question. SDT states that human beings universally require ongoing satisfaction of three basic psychological needs to function optimally - autonomy, competence, and relatedness. ${ }^{15}$ Autonomy is the need for volition and psychological freedom from outside pressures or coercion; competence is the need to feel effective and capable of success in a given domain (e.g., with one's health); and relatedness is the need for belongingness and to feel connected with significant others. ${ }^{15}$ Accordingly, SDT's perspective is that environments which support these basic needs (and autonomy in particular) will yield greater autonomous motivation, engagement, and wellness, whereas environments that thwart them will provoke the opposite - apathy, maladjustment, stress, and psychopathology. ${ }^{15}$ Relationships motivation theory (RMT) - a key minitheory within SDT in which the present study is grounded - focuses on basic psychological needs in interpersonal relationships. In brief, it posits that relatedness is an intrinsic need that inclines people to be volitionally engaged in relationships, and that receiving more autonomy support from a relational partner facilitates the receiver's need satisfaction, sense of secure attachment, and wellness. ${ }^{15}$

In a healthcare context, studies show that when patients perceive their physicians as more autonomy-supportive, it leads to a host of beneficial outcomes, including better diabetes self-management, medication adherence, and quality of life. ${ }^{16,17,18}$ Other studies are complimentary, showing that when patients perceive more autonomy support from their family doctors and care team, it leads to better outcomes with smoking cessation and abstinence, exercise, and diet-related changes.19,20 In medical education, autonomy-supportive work environments have been linked to greater work engagement, less work-related burnout, and higher well-being. ${ }^{21}$ Hence, the benefits of autonomy-supportive healthcare climates are innumerable. ${ }^{22}$ Despite this, however, the dynamics of basic psychological needs and patient-provider relationships have not been studied in the emerging sphere of telehealthcare - where barriers to meeting autonomy, competence, and relatedness needs seem worryingly present.

Through a RMT lens, it stands to reason that virtual versus in-person healthcare climates might be perceived as less autonomysupportive (and therefore motivating) to patients. Not being able to physically see one's physician and care team - to read their verbal and non-verbal cues, to experience their touch, or to plainly recognize that their constructive suggestions are sincere simply creates for less relatedness. This may then prevent patients from meeting their need for competence - for example, out of hesitation to open-up about personal struggles in the face of less inviting or familiar (and thereby psychologically safe) virtual territory. Many patients are also relatively new to and uncertain about virtual medicine (e.g., with respect to trusting and effectively navigating the process), which may inhibit their sense of autonomy and freedom to express themselves, in favour of taking their care provider's lead. In other words, the very nature of virtual healthcare - while convenient in many cases - seems to threaten

(c) The Internet Journal of Allied Health Sciences and Practice, 2022 
patients' psychological needs for optimal relationships with their care providers: aspects that might translate into scarcely, partially, or fully engaging with healthcare suggestions and autonomous self-control. ${ }^{23}$

Though there are surely benefits to tele-healthcare (e.g., flexibility in scheduling, administrative cost-savings, and potentially convenience), and it will undoubtedly continue to grow in popularity, we argue that its potential barriers to psychological need satisfaction are worthy considerations, chiefly because obstacles to meeting these basic needs are known to come at significant functional costs - to engagement, healthy relationships, and long-term health and wellness outcomes. ${ }^{24,25}$ Additionally, in a healthcare field already plagued by distress and burnout, we believe it is imperative to consider not just patients' psychological needs in virtual care, but also the basic psychological needs of healthcare providers. ${ }^{26,27}$

The present study aims to address part of this question by investigating how telephone versus in-person healthcare experiences might differentially impact patients' perceived autonomy support and corresponding relationship needs satisfaction with their family doctor and care team. To our knowledge, there are no published studies examining these specific matters, neither within the Patient Medical Home (PMH) context of family medicine, nor within other healthcare specialties that may utilize virtual modalities of clinical care (e.g., psychology, pharmacy, physiotherapy, social work, nursing, etc.).

\section{Guided by RMT, our hypotheses are as follows:}

1. Patients' perceived autonomy support (within the tele-health and in-person setting) will positively relate to their relationship needs satisfaction (autonomy, competence, and relatedness) with their family doctor and PMH care team.

2. Compared to patients visiting their family doctor and care team in person, patients visiting by telephone will perceive lower levels of autonomy support.

\section{METHODS}

\section{Participants \& Procedure}

A total of 104 patients (48 who visited in person and 56 who visited virtually) were invited to take part in this study. Inclusion criteria required that age was between 18-99 years, that they could speak English (or at least have someone who could translate for them), and that they had an active email address. There were no specific recruitment methods beyond verbally asking patients if they were interested in participating, and then following up with an email invitation. To prevent participant identification and mitigate response bias, surveys were completely anonymous. Therefore, reminder emails were not sent out, and whether patients participated or not could have no bearing on their standing in the clinic.

Email invitations contained an ethics-approved consent form, information about the nature of the study, and a link to one of two survey versions, based on each type of visit: virtual or in-person. Patients who visited by telephone were therefore sent the "virtual" version of the survey, while those who visited in person were sent the "in-person" version of the survey. Both internet-based surveys contained various sociodemographic questions, as well as several previously validated scales, which are freely available for research purposes (and therefore did not require any special permissions - see Measures).

Of note, there were 6 different physicians involved in this study - each of whom were seeing roughly equal amounts of patients by telephone and in person. For these physicians, scheduling virtual visits involves 3 primary factors: patient choice (likely the predominant factor), scheduling directions provided to reception (applies to all physicians), and physician review of schedule in the morning (may suggest change in virtual or in-person visit, based on presenting complaint). Thus, the decision of visit type in this study (i.e., telephone vs. in person) was technically tied to each physician, but in a way that typically reflected the nature of the presenting issue, and that involved a great deal of patient choice (i.e., to self-select into telephone or clinic visits).

\section{Ethical Approval}

This study was approved by the University of Calgary Conjoint Health Research Ethics Board - REB \#20-1471.

\section{Measures}

Each survey started with several sociodemographic questions to help us understand the sample makeup. These included the following: "What is your gender?" (Male, female, non-binary, transgender, not listed, prefer not to answer); "What is your ethnicity?" (Asian, Black, Caucasian, Indigenous, Hispanic, prefer not to answer); "What is your highest level of education?" (No formal education, high school diploma, college degree, vocational training, bachelor's degree, master's degree, professional degree, doctoral degree, other); "What is your current work status?" (Employed full-time, employed part-time, self-employed/freelance, interning, studying, unemployed - looking for work, unemployed - not looking for work, homemaker, military/forces, retired, not able to work, other); "What is your household income?" (Under \$20,000, \$20,001-40,000, \$40,001-60,000, \$60,001-80,000, $\$ 80,001-100,000$, and $\$ 100,001$ and over); "What is the length of time you have known your family physician?" (Less than 1 year,

(c) The Internet Journal of Allied Health Sciences and Practice, 2022 
1-5 years, $6-10$ years, 11-15 years, 16-20 years, 20+ years); "What was the main reason for your recent visit with your family physician?" (physical health, mental health, medications, forms/documents, other); and "What is your preference for medical visit type?" (Virtual or in person). Following these questions, patients completed the following two measurement instruments, derived from SDT.

\section{Healthcare Climate Questionnaire}

This 6-item questionnaire measures a person's perceived level of autonomy support within the healthcare domain. ${ }^{28}$ The wording of the stem was modified to reflect patients' virtual (i.e., telephone) or in-person encounters visiting their family doctor and care team at the University of Calgary's South Health Campus Family Medicine Teaching Clinic. Since this multidisciplinary clinic is a Patient Medical Home (i.e., often involving a medical office assistant and/or others, such as a clinical pharmacist, dietician, or social worker), the scale was adapted to reflect the interpersonal climate of the overall clinic experience, rather than solely with their family doctor. Using a Likert-type scale (where $1=$ strongly disagree, $4=$ neutral, and $7=$ strongly agree), patients rated their level of agreement with each statement, based on their most recent virtual or in-person visit to the clinic. Example statements included: "I feel that my provider(s) has provided me choices and options," "I feel understood by my provider(s)," "My provider(s) conveys confidence in my ability to make changes," and "My provider(s) listens to how I would like to do things." This scale has demonstrated strong psychometrics properties. ${ }^{29}$

\section{Basic Need Satisfaction in Relationships Questionnaire}

This 9-item questionnaire measures a person's level of basic psychological need satisfaction (autonomy, competence, relatedness) within their relationship with someone - in this case, their family doctor and care team. ${ }^{30}$ The stem was adapted to read: "In my relationship with my health care provider(s) at the South Health Campus Family Medicine Teaching Clinic..." This stem was identical for both virtual and in-person groups; items were simply tailored slightly, to reflect the type of visit. For example, statements for the in-person visit included: "When I am with my provider(s), I feel free to be who I am," "When I am with my provider(s), I have a say in what happens and I can voice my opinion," and "When I am with my provider(s), I often feel a lot of distance in our relationship." Conversely, example items in the virtual survey included: "When I have virtual visits with my provider(s), I feel loved and cared about," "When I have virtual visits with my provider(s), I feel very capable and effective," and "When I have virtual visits with my provider(s), I feel controlled and pressured to be certain ways." Patients rated each statement on a Likert-type scale, indicating how "true" each was for them (where $1=$ not at all true, $4=$ somewhat true, and $7=$ very true). Items formed three subscales (one for each need), which were averaged together to form an aggregated needs satisfaction in relationships score. This scale has also been shown to have good reliability in prior studies..$^{30}$

\section{Statistical Analyses}

The survey software Qualtrics ${ }^{\mathrm{TM}}$ was used to create, disseminate, and export all patient-related data. We then used the software program SPSS v.25.0TM for all our statistical analyses. There were no incomplete surveys (under half of scales completed), so all participant data were analyzed. All data were then standardized and deemed to be normally distributed. The basic needs (autonomy, competence, and relatedness) in relationships subscales were aggregated into a total mean score for basic needs satisfaction, as in prior studies. ${ }^{31,32}$ Cronbach's alpha coefficients were calculated and indicated good preservation of reliability for all scales (all > . 82). Descriptive statistics included a breakdown of the "virtual" and "in-person" patient sample characteristics (see Measures). Of note, this breakdown (see Table 1) includes actual item responses only, and not survey items that patients did not select. Variable relationships were then assessed using Pearson correlation, which showed no evidence of multicollinearity. These data informed several subsequent regression analyses, using $95 \%$ confidence intervals, which assessed the impact of patients' perceptions of autonomy support (i.e., the healthcare climate) on their relationship needs satisfaction with their family doctor and care team. Standardized beta coefficients, estimates of model fit, and $p$-values are presented accordingly.

Since the nesting of patients within physicians (i.e., multiple patients linked to a single physician) created the possibility that the general independent observations assumption of statistical procedures would be violated, we used a random effects regression design to examine our research questions. For each patient group (coded into $1=$ virtual or $2=$ in-person), perceived autonomy support was entered as the random effects (i.e., predictor) variable, aggregated relationship needs satisfaction was entered as the dependent variable, and the sociodemographic factors (listed above) were entered as the fixed effects variables. Following this, a univariate analysis of variance (ANOVA) compared the virtual vs. in-person groups in their perceived autonomy support, adjusting for any covariate effects of the sociodemographic factors. Pairwise comparisons then compared each group's estimated marginal mean scores in perceived autonomy support, using a Bonferroni $p$-value correction. Partial eta squared $\left(\eta_{p}^{2}\right)$ values are provided as estimates of the standardized effect size (as per Cohen's $d$ effect sizes, where: $0.2,0.5$, and 0.8 are considered small, medium, and large, respectively), along with values for the $F$-test(s), mean square error (MSE), and $p$ statistic.

(c) The Internet Journal of Allied Health Sciences and Practice, 2022 


\section{RESULTS}

\section{Sample Characteristics}

Out of the 104 patients that were invited to partake in this study, data from 66 were analyzed: $34(52 \%)$ that had visited in person and $32(48 \%)$ that had visited virtually. The mean age in the "in-person" arm was $M=47.8$ years $(S D=8.2)$ with a range of 19-76 years. Meanwhile, the mean age in the "virtual" arm was $M=48.9$ years $(S D=8.6)$ with a range of $18-87$ years. Overall, $58 \%$ of the total sample reported a preference for in-person over virtual visits (where $68.8 \%$ of patients who visited in person reported preferring "in-person" visits and $47.1 \%$ of patients who visited by telephone reported preferring "virtual" visits). The mean relationship needs satisfaction scores for telephone visits were as follows: autonomy $(M=4.67, S D=0.65)$, competence $(M=$ 4.52, $S D=0.69)$ and relatedness $(M=4.14, S D=0.69)$. These scores were similar for the in-person group: autonomy $(M=4.79$, $S D=0.40)$, competence $(M=4.66, S D=0.48)$, and relatedness $(M=4.28, S D=0.68)$. Further sample characteristics for both groups are presented in Table 1.

\section{Variable Relationships}

The correlational analyses revealed that patients' perceived autonomy support positively related to their relationship needs satisfaction with their family doctor and care team, both among patients who visited in person $(r=.625, p<.001)$ and virtually $(r=$ $.566, p=.001$ ). These findings informed two corresponding random effects regression models (one for both the virtual and inperson groups), which each controlled for the full range of sociodemographic factors (see Statistical Analyses). As was hypothesized based on RMT, these confirmed a strong positive association between patients' perceived autonomy support and relationship needs satisfaction with their family doctor and care team, both within the in-person $(\beta=.441, p=.013)$ and virtual $(\beta$ $=.450, p=.048)$ clinical settings. The overall model fits were $R^{2}=.570$ and $R^{2}=.505$, respectively.

\section{Patient Autonomy in Virtual vs. In-Person Healthcare Encounters}

Next, we sought to determine whether differences existed, in perceived autonomy support, between patients who visited their family doctor and care team in person versus virtually. First, however, we examined our sample's characteristics, which indicated that both groups were different in several ways. For instance, more individuals in the telephone versus in-person group were receiving care that was inconsistent with their preference (approx. 53\% vs. 32\%). The telephone group was also lower in income (approx. $60 \%$ of the group made over $60 \mathrm{k}$ versus $81 \%$ in the in-person group), and it was more likely that their visit was related to medications, compared to the in-person group (approx. $35 \%$ versus 19\%). In addition, about $25 \%$ of patients in the in-person group had been seeing their physician for under 1 year. Since we felt that these differences could influence perceived autonomy support, we conducted a univariate ANOVA with group (virtual or in-person) entered as the random factor (i.e., predictor), perceived autonomy support entered as the dependent variable, and all sociodemographic factors (see Measures) entered as covariates.

Perhaps unsurprisingly, the ANOVA showed that patients' preference for visit type (although no other sociodemographic factor) was a significant predictor of their perceived autonomy support, $F(1,51)=6.38, M S E=27.82, p=.015, \eta^{2}=.11$. However, even after adjusting for this and the various other sociodemographic factors, the mean difference in perceived autonomy support, between the in-person $(M=6.28, S E=.16)$ versus virtual $(M=5.75, S E=0.17)$ patient groups, was still statistically significant, $F$ $(1,51)=4.87, M S E=28.82, p=.032, \eta_{p}^{2}=.09$. The corresponding effect sizes of visit preference and group (telephone vs. inperson) on perceived autonomy support were therefore considered small.

Table 1 - Sample characteristics for telephone vs. in-person patient groups

\begin{tabular}{lcc} 
& \multicolumn{2}{c}{ Frequency (\%) of cases by group } \\
\cline { 2 - 3 } Characteristics & In-Person $(\mathbf{n}=34)$ & Telephone $(\mathbf{n}=\mathbf{3 2})$ \\
\hline Gender & $11(34.4)$ & $9(26.5)$ \\
Male & $21(65.6)$ & $22(64.7)$ \\
Female & & \\
Ethnicity & $26(81.3)$ & $28(82.4)$ \\
Caucasian & $4(12.5)$ & $1(2.9)$ \\
Asian & $2(6.3)$ & $2(5.9)$ \\
Hispanic & $0(0)$ & $2(5.9)$ \\
Prefer not to answer & & $1(2.9)$ \\
Highest level of education & $0(0)$ & $6(17.6)$ \\
No formal education & $6(18.8)$ & $5(14.7)$ \\
High school diploma & $3(9.4)$ & $3(8.8)$ \\
College degree & $8(25.0)$ & \\
Vocational training & &
\end{tabular}

(c) The Internet Journal of Allied Health Sciences and Practice, 2022 


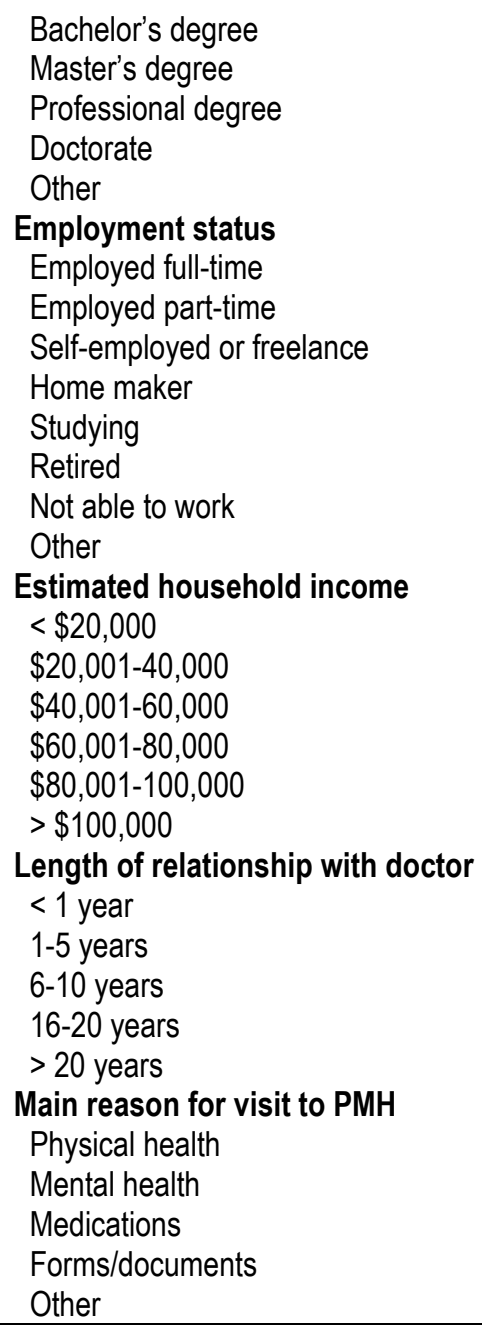

\section{DISCUSSION}

In the present study, we set out to determine the relationship between patients' perceptions of the healthcare climate (i.e., of physician autonomy support) and their relationship needs satisfaction (autonomy, competence, and relatedness) with their family doctor and care team. Additionally, we explored whether patients' perceptions of autonomy support differed based on the visit being virtual versus in person. Interestingly, we found that patients' perceived autonomy support not only strongly predicted how needs-satisfying their relationship was with their care provider(s), but that it was significantly lower among patients who visited their clinic virtually (by telephone) versus in person. In this section, we briefly discuss the potential explanations for these findings, along with their implications for healthcare providers and patients.

According to SDT, perceived autonomy functions as the anchor for satisfaction of the three basic psychological needs for optimal motivation and wellness. ${ }^{15}$ Our findings support this notion, in that patients' perceived autonomy support was pivotal to their relationship needs satisfaction with their care provider(s). In other words, the more that patients found their provider(s) to be humanistic (e.g., in taking their perspectives into account, encouraging questions and choices, positioning information in noncontrolling and informational ways, and incorporating shared decision making), the more fulfilling they found their patient-provider relationship. Our findings also support RMT's postulates, more specifically, that perceived autonomy fosters a greater sense of relational competence or efficacy (e.g., in understanding health-related suggestions, feeling capable of success and of being able to overcome challenges, and not feeling like a failure if or when setbacks occur) and relatedness (feeling connected, understood, and valued by the care team, and sensing a sincere and non-judgmental environment).

While meeting patients' medical needs may be feasible during virtual versus in-person visits, our results suggest that supporting their autonomy may in fact be harder in the virtual setting. From a self-determination standpoint, it could be that telephone medicine is devoid of typical environmental (e.g., doctor's office) and care provider (e.g., body language) cues, which make it more difficult 
for patients to engage and feel engaged. This lack of relatedness may then create a higher cognitive load, given the inevitable need for patients to rely on the caller's tone and prosody of speech to process and interpret information, thereby threatening their ability to remain present, and subsequently, their perceived competence. Take a scenario in which a physician challenges a patient to try to encourage them to take more personal responsibility for their health: not being able to see the physician may provoke more distraction, tension, or pressure to sound a certain way (e.g., less defensive), compared to a visit in person, where constructive criticisms are typically accompanied by positive social cues and gestures of warmth (e.g., touch, facial expression, empathy). Studies showing that a physician's touch is a powerful form of non-verbal communication for human connection, and that tone of voice in motivating others is just as important as the message itself, both support this notion. ${ }^{33,34}$ Coupled with reduced certainty around the typical routine or desirability of telephone visits, patients may also experience internal conflicts about whether to reveal-versus-hide their true thoughts and feelings, even if they disagree with the physician.

Interestingly, while between-group differences in perceived autonomy support were evident regardless of sociodemographic sample inequivalences, findings suggest that a larger proportion of virtual patients had a visit that was inconsistent with their preference (i.e., for in-person visits), and that this likely reduced their perceptions of autonomy support. This leads us to consider whether more patients that self-selected into a virtual (versus in-person) visit might have done so for less autonomous reasons (e.g., due to safety concerns related to the COVID-19 pandemic). There may also be other plausible explanations for the betweengroup difference we found in perceived autonomy support that this study did not account for. For instance, RMT tells us that when a person (e.g., a patient) senses that their relational partner (e.g., a physician) lacks autonomy for connecting within the relationship, or when one's needs for relatedness and autonomy are turned against each other (e.g., in situations where gaining relatedness comes at the cost of losing autonomy), it will tend to undermine relationship needs satisfaction (and therefore feelings of authenticity, emotional reliance, transparency, and non-defensiveness). ${ }^{15} \mathrm{It}$ therefore follows that if physician motivation towards using tele-healthcare was felt to be non-volitional (i.e., pressured) - either by physicians themselves and/or by patients alike - that it too could constrain both party's sense of autonomy and wellness. Whether this is true, and how this might change once the pandemic and its restrictions settle, are topics for future research.

Importantly, these tangible limitations are inherent in any type of telephone healthcare, regardless of how close or longstanding the relationship between patient and doctor. It therefore follows that the differences in perceived autonomy support in the present study (which were found among patients whose physicians typically know them well - in other words, who appreciate the specific patient context in a long-standing, continuous, therapeutic relationship) would be more pronounced between doctors and patients who know each other less, or not at all. The recent rise of commercial third-party healthcare companies, offering walk-in style virtual medicine, "where and when it's convenient for you," undermines the longitudinal relationship and rapport that family physicians and care teams form with their patients. Commercial entities argue this technology is revolutionizing access to healthcare and alleviating pressures on the system. Doctors, on the other hand, are concerned that these types of platforms are not filling a medical care gap, but creating more cracks instead. ${ }^{35} \mathrm{It}$ is therefore critical to consider not just the medical needs of patients and how cost-saving or convenient virtual care might be, but also the barriers this type of care may create for patients, in meeting their basic psychological needs. With ample evidence that thwarting these needs leads to poorer health and wellness outcomes, we recommend heeding SDT's suggestions and not taking these preliminary findings lightly.

With that cautionary statement, however, we are not arguing that virtual medicine is inferior, nor that all patient visits should be conducted in person. In fact, we agree with others that virtual medicine can potentially bolster our ability to be patient-centered, at least in some situations. $.7,7,9$ We are simply illustrating how virtual visits may be innately more challenging in terms of supporting patients' humanistic needs for self-determination. If true, our findings that virtual healthcare encounters are typically perceived as less autonomy-supportive suggests that more research is needed to determine how interventions, such as educational training, could help physicians and other allied healthcare providers to overcome this obstacle. At present, most healthcare providers, including physicians, receive little or no training in psychology (such as how to support and avoid constraining others' autonomy, competence, and relatedness needs), nor in approaches to optimizing virtual care provision. As studies on the role of psychological theory (including SDT) in integrated healthcare suggest, this would greatly benefit patients, especially in settings involving the telephone. ${ }^{36,37,38,39}$ Our results suggest that, without this knowledge and awareness, virtual patients' engagement and patientprovider relationship may stand to suffer.

A final point of concern with much of the emerging research suggesting that virtual care is both feasible, and perhaps even preferential, is the validity evidence being used to form that conclusion. Many studies (of which we only mention a few) have partially or fully relied on patient and/or physician "satisfaction" surveys, which were either created de novo and/or were not derived from any established theoretical frameworks. $40,41,42,43$ The inherent problem with this is that favourable ratings can create a false sense of confidence in virtual healthcare, without leading to any of the health and wellness outcomes we believe or want them to. Clear evidence of this is seen in the field of education, where studies repeatedly show that learner satisfaction and positive teacher

(C) The Internet Journal of Allied Health Sciences and Practice, 2022 
evaluations do not actually translate into better learning outcomes. $44,45,46$ Hence, we feel that it is imperative to draw on empirically supported frameworks like SDT, with well-established scales and good validity evidence, if we want to reliably study and understand the true benefits and limitations of virtual healthcare, for all those involved.

\section{Limitations, Strengths, and Future Directions}

This study has several noteworthy limitations which may help guide future research. First, this was a single-center pilot project that included a relatively low sample size (i.e., in the virtual and in-person groups), albeit with a response rate of $63.5 \%$. Additionally, data came from patients visiting a family medicine teaching clinic (otherwise known as a Patient Medical Home), situated within a new hospital in a relatively affluent area of the city of Calgary ${ }^{47} \mathrm{As}$ can be seen in this study's sample characteristics, the majority of the patients could be considered middle-to-upper class, socioeconomically. The patient sample was also predominantly Caucasian, and there were more females than males who responded to each survey. The generalizability of our findings to other populations is therefore uncertain. That said, we gathered at least 30 surveys per group, to ensure that the distribution of our sample means would approximate statistical normality. Furthermore, the study was sufficiently powered to detect significant group differences, and the non-negligible effect size suggests true motivational-psychological differences between the virtual and inperson healthcare environments. Further research is nonetheless needed to corroborate and extend this research, including among other clinics, socioeconomic tiers, and patient populations.

Secondly, this study relied on convenience sampling and results are derived from self-report surveys, which can be subject to bias. For example, there was some potential for selection bias in how or when physicians decided which patients to recruit, as well as the potential for different physician styles (i.e., in providing autonomy support) to influence results. Additionally, while patient data was anonymous, information provided about the survey may have swayed patients' responses. We attempted to mitigate these limitations by advising physicians, a priori, that patient invitations should be offered regularly and independently of visit type (e.g., medication refills, physical ailments, mental health concerns, forms/documents, meet and greets, etc.), provided it was felt to be appropriate (i.e., discounting serious acute illnesses, tragedies, or significant interference with clinic scheduling). Additionally, we collected data from six different physicians, and because patients were nested within these physicians, we used random effects modelling, to satisfy the assumption of physician independence. Finally, we collected data from a wide variety of patients (different ages, backgrounds, visit-related reasons, and preferences for visit type). This way, the sample data would best represent the general patient population from our PMH panels.

A third limitation of the present study is that its virtual component focused solely on telephone medicine and not videoconferencing, which presumably could lead to different results. This was because telephone visits are normally the standard non-in-person visit type for most family medicine clinics, compared to using potentially less equitable and more time-consuming video technologies, which many patients struggle with and/or may not have access to. Future studies may therefore explore this avenue, in terms of whether video-related visits are felt to be as autonomy-supportive as in-person visits. This study does not address the issue of disparities in access to virtual healthcare (i.e., for patients who cannot use or afford telephones or computers), but this too is considered a real issue in need of further address. 48

A few final points of mention are that, in a PMH model of healthcare, the environment is an academic teaching facility where visits are predominantly led by a resident physician. Thus, some patients may not actually have seen or spoken with their family physician, directly. This may have rendered certain survey questions (e.g., length of time knowing the family physician) less relevant to patients and, relatedly, may skew the interpretation of our results, pertaining to their ratings of autonomy support. Additionally, while not a limitation per se, one of the physician authors involved in the patient visits and recruitment for this study (AN) is immersed in SDT research. Hence, his awareness and regular practice of trying to be autonomy-supportive with patients likely influenced results, although in a positive way. In view of this, the fact that the standardized difference between the virtual and inperson groups' perceived autonomy support was still sizeable, and that this study was conducted within a PMH environment (which is designed to be collaborative, patient-centered, and emphasizing of a longitudinal therapeutic relationship) suggests that, if anything, findings from the present study are probably an underestimate of the mean difference between visit types within the general population.

\section{CONCLUSIONS}

Findings from this research suggest that, while virtual care may be useful and potentially necessary to safely meet patients' medical needs during the time of COVID-19, it may also be sub-optimal for fostering their autonomy and patient-provider relationship. Albeit preliminary, these results have potentially broad and significant implications for the delivery of healthcare, not just in family medicine but beyond. We therefore urge other healthcare workers and researchers to join us in studying this phenomenon more carefully, especially given the inevitable move towards continued virtual care in the future. From a SDT perspective, let us not ignore the

(c) The Internet Journal of Allied Health Sciences and Practice, 2022 
vital psychological needs that patients and care providers have for optimal engagement within their healthcare system - their motivation, health, and well-being depend on it.

\section{REFERENCES}

1. Spelman JF, Brienza R, Walsh RF, et al. A Model for Rapid Transition to Virtual Care, VA Connecticut Primary Care Response to COVID-19. J Gen Intern Med. Published online 2020. doi:10.1007/s11606-020-06041-4

2. Rosen CB, Joffe S, Kelz RR. COVID-19 Moves Medicine into a Virtual Space: A Paradigm Shift From Touch to Talk to Establish Trust. Ann Surg. Published online 2020. doi:10.1097/SLA.0000000000004098

3. Kohan L, Durbhakula S, Zaidi M, et al. Changes in Pain Medicine Training Programs Associated with COVID-19. Anesth Analg. Published online 2020. doi:10.1213/ane.0000000000005314

4. Zhou X, Snoswell CL, Harding LE, et al. The Role of Telehealth in Reducing the Mental Health Burden from COVID-19. Telemed e-Health. Published online 2020. doi:10.1089/tmj.2020.0068

5. Mammen JR, Elson MJ, Java JJ, et al. Patient and physician perceptions of virtual visits for Parkinson's disease: A qualitative study. Telemed e-Health. Published online 2018. doi:10.1089/tmj.2017.0119

6. Roy B, Nowak RJ, Roda R, et al. Teleneurology during the COVID-19 pandemic: A step forward in modernizing medical care. J Neurol Sci. Published online 2020. doi:10.1016/j.jns.2020.116930

7. $\quad$ Uscher-Pines L, Sousa J, Raja P, Mehrotra A, Barnett ML, Huskamp HA. Suddenly becoming a "Virtual doctor": Experiences of psychiatrists transitioning to telemedicine during the COVID-19 pandemic. Psychiatr Serv. Published online 2020. doi:10.1176/APPI.PS.202000250

8. Olwill C, Mc Nally D, Douglas L. Psychiatrist Experience of Remote Consultations by Telephone in an Outpatient Psychiatric Department during the Covid - 19 Pandemic. Ir J Psychol Med. Published online 2020. doi:10.1017/ipm.2020.51

9. Lamplot JD, Pinnamaneni S, Swensen-Buza S, et al. The Virtual Shoulder and Knee Physical Examination. Orthop J Sport Med. Published online 2020. doi:10.1177/2325967120962869

10. Yu JC, McIntyre M, Dow H, Robinson L, Winston P. Changes to rehabilitation service delivery and the associated physician perspectives during the COVID-19 pandemic: A mixed-methods needs assessment study. Am J Phys Med Rehabil. Published online 2020. doi:10.1097/PHM.0000000000001516

11. Bertman S. Pursuing Humanistic Medicine in a Technological Age. J Patient Exp. Published online 2017. doi:10.1177/2374373517699269

12. Simpkin AL, Dinardo PB, Pine E, Gaufberg E. Reconciling technology and humanistic care: Lessons from the next generation of physicians. Med Teach. Published online 2017. doi:10.1080/0142159X.2017.1270434

13. Fischer M, Brown-Johnson CG, Safaeinili N, et al. Humanism in Telemedicine: Connecting through Virtual Visits during the COVID-19 Pandemic.; 2020.

14. Kelly MA, Gormley GJ. In, but out of touch: Connecting with patients during the virtual visit. Ann Fam Med. 2020;18(5). doi:10.1370/afm.2568

15. Ryan RM, Deci EL. Self-Determination Theory: Basic Psychological Needs in Motivation Development and Wellness. Guilford Publishing; 2017.

16. Williams GC, McGregor HA, Zeldman A, Freedman ZR, Deci EL. Testing a Self-Determination Theory Process Model for Promoting Glycemic Control Through Diabetes Self-Management. Heal Psychol. Published online 2004. doi:10.1037/0278-6133.23.1.58

17. Williams GC, McGregor HA, King D, Nelson CC, Glasgow RE. Variation in perceived competence, glycemic control, and patient satisfaction: Relationship to autonomy support from physicians. Patient Educ Couns. Published online 2005. doi:10.1016/j.pec.2004.04.001

18. Williams GC, Patrick H, Niemiec CP, et al. Reducing the health risks of diabetes: How self-determination theory may help improve medication adherence and quality of life. Diabetes Educ. Published online 2009. doi:10.1177/0145721709333856

19. Williams GC, Minicucci DS, Kouides RW, et al. Self-determination, smoking, diet and health. Health Educ Res. Published online 2002. doi:10.1093/her/17.5.512

20. Williams GC, Patrick H, Niemiec CP, Ryan RM, Deci EL, Lavigne HM. The Smoker's Health Project: A selfdetermination theory intervention to facilitate maintenance of tobacco abstinence. Contemp Clin Trials. 2011;32(4):535543. doi:10.1016/j.cct.2011.03.002

21. Kusurkar RA, Croiset G. Autonomy support for autonomous motivation in medical education. Med Educ Online. Published online 2015. doi:10.3402/meo.v20.27951

22. Entwistle VA, Carter SM, Cribb A, McCaffery K. Supporting patient autonomy: The importance of clinician-patient

(C) The Internet Journal of Allied Health Sciences and Practice, 2022 
relationships. J Gen Intern Med. Published online 2010. doi:10.1007/s11606-010-1292-2

23. Williams GC, McGregor HA, Sharp D, et al. Testing a self-determination theory intervention for motivating tobacco cessation: Supporting autonomy and competence in a clinical trial. Heal Psychol. Published online 2006. doi:10.1037/0278-6133.25.1.91

24. Ryan R, Deci E. Self-determination theory and the facilitation of intrinsic motivation. Am Psychol. 2000;55(1):68-78. doi:10.1037/0003-066X.55.1.68

25. Teixeira PJ, Marques MM, Silva MN, et al. A Classification of Motivation and Behavior Change Techniques Used in SelfDetermination Theory-Based Interventions in Health Contexts. Motiv Sci. Published online 2020. doi:10.1037/mot0000172

26. Dyrbye LN, Thomas MR, Shanafelt TD. Systematic review of depression, anxiety, and other indicators of psychological distress among U.S. and Canadian medical students. Acad Med. 2006;81(4):354-373. doi:10.1097/00001888200604000-00009

27. Shanafelt TD, Hasan O, Dyrbye LN, et al. Changes in Burnout and Satisfaction with Work-Life Balance in Physicians and the General US Working Population between 2011 and 2014. Mayo Clin Proc. Published online 2015. doi:10.1016/j.mayocp.2015.08.023

28. Williams GC, Cox EM, Kouides R, Deci EL. Presenting the facts about smoking to adolescents: Effects of an autonomysupportive style. Arch Pediatr Adolesc Med. Published online 1999. doi:10.1001/archpedi.153.9.959

29. Williams GC, Grow VM, Freedman ZR, Ryan RM, Deci EL. Motivational Predictors of Weight Loss and Weight-Loss Maintenance. J Pers Soc Psychol. Published online 1996. doi:10.1037/0022-3514.70.1.115

30. La Guardia JG, Ryan RM, Couchman CE, Deci EL. Within-person variation in security of attachment: A selfdetermination theory perspective on attachment, need fulfillment, and well-being. J Pers Soc Psychol. Published online 2000. doi:10.1037/0022-3514.79.3.367

31. Neufeld A, Malin G. How medical students' perceptions of instructor autonomy-support mediate their motivation and psychological well-being. Med Teach. Published online 2020. doi:10.1080/0142159X.2020.1726308

32. Chen B, Vansteenkiste M, Beyers W, et al. Basic psychological need satisfaction, need frustration, and need strength across four cultures. Motiv Emot. 2015;39(2):216-236. doi:10.1007/s11031-014-9450-1

33. Kelly M, Svrcek C, King N, Scherpbier A, Dornan T. Embodying empathy: A phenomenological study of physician touch. Med Educ. Published online 2020. doi:10.1111/medu.14040

34. Weinstein N, Zougkou K, Paulmann S. You "have" to hear this: Using tone of voice to motivate others. J Exp Psychol Hum Percept Perform. Published online 2018. doi:10.1037/xhp0000502

35. Fraser H, Coiera E, Wong D. Safety of patient-facing digital symptom checkers. Lancet. Published online 2018. doi:10.1016/S0140-6736(18)32819-8

36. Kenkel MB, Deleon PH, Mantell EO, Steep AE. Divided no more: Psychology's role in integrated health care. Can Psychol. 2005;46(4). doi:10.1037/h0087026

37. Gambling T, Long AF. Exploring patient perceptions of movement through the stages of change model within a diabetes tele-care intervention. J Health Psychol. 2006;11(1). doi:10.1177/1359105306058854

38. Murray A, Hall AM, Williams GC, et al. Effect of a self-determination theory-based communication skills training program on physiotherapists' psychological support for their patients with chronic low back pain: A randomized controlled trial. Arch Phys Med Rehabil. 2015;96(5). doi:10.1016/.apmr.2014.11.007

39. Chemtob K, Rocchi M, Arbour-Nicitopoulos K, Kairy D, Fillion B, Sweet SN. Using tele-health to enhance motivation, leisure time physical activity, and quality of life in adults with spinal cord injury: A self-determination theory-based pilot randomized control trial. Psychol Sport Exerc. 2019;43. doi:10.1016/j.psychsport.2019.03.008

40. Pflugeisen BM, Mou J. Patient Satisfaction with Virtual Obstetric Care. Matern Child Health J. 2017;21(7). doi:10.1007/s10995-017-2284-1

41. Miner H, Fatehi A, Ring D, Reichenberg JS. Clinician Telemedicine Perceptions During the COVID-19 Pandemic. Telemed e-Health. Published online 2020. doi:10.1089/tmj.2020.0295

42. Murphy RP, Dennehy KA, Costello MM, et al. Virtual geriatric clinics and the COVID-19 catalyst: A rapid review. Age Ageing. 2020;49(6). doi:10.1093/ageing/afaa191

43. Lun R, Walker G, Daham Z, et al. Transition to virtual appointments for interventional neuroradiology due to the COVID19 pandemic: A survey of satisfaction. J Neurointerv Surg. 2020;12(12). doi:10.1136/neurintsurg-2020-016805

44. Spooren P, Brockx B, Mortelmans D. On the Validity of Student Evaluation of Teaching: The State of the Art. Rev Educ Res. 2013;83(4). doi:10.3102/0034654313496870

45. Uttl B, White CA, Gonzalez DW. Meta-analysis of faculty's teaching effectiveness: Student evaluation of teaching ratings and student learning are not related. Stud Educ Eval. 2017;54. doi:10.1016/j.stueduc.2016.08.007

46. Hornstein HA. Student evaluations of teaching are an inadequate assessment tool for evaluating faculty performance. Cogent Educ. 2017;4(1). doi:10.1080/2331186X.2017.1304016

(C) The Internet Journal of Allied Health Sciences and Practice, 2022 
47. Gwynne MD, Daaleman TP. Patient-centered medical home. In: Chronic Illness Care: Principles and Practice. ; 2018. doi:10.1007/978-3-319-71812-5_28

48. Ortega G, Rodriguez JA, Maurer LR, et al. Telemedicine, COVID-19, and disparities: Policy implications. Heal Policy Technol. Published online 2020. doi:10.1016/j.hlpt.2020.08.001 